\title{
SERVICIOS ECOSISTÉMICOS PROVISTOS POR LOS SUELOS EN UNA CUENCA DE SANTA FE, ARGENTINA
}

\section{ECOSYSTEM SERVICES PROVIDED BY SOILS IN A BASIN OF SANTA FE, ARGENTINA}

\author{
Sergio Montico ${ }^{(*)}$, Néstor Di Leo, Beatriz Bonel y José Berardi \\ Facultad de Ciencias Agrarias, Universidad Nacional de Rosario. Zavalla, Santa Fe, Argentina. \\ ${ }^{(*)}$ e_mail: smontico@unr.edu.ar
}

\begin{abstract}
RESUMEN
Los suelos forman parte del ecosistema y brindan servicios múltiples que no son convenientemente ponderados por la sociedad. El objetivo de este trabajo es evaluarlos en una cuenca rural, con el propósito de identificar y valorar las funciones y beneficios ambientales, sociales, culturales y económicos que brindan. En la cuenca del arroyo Ludueña en el sur de Santa Fe (Argentina), se aplicó una metodología de valoración de la potencia de los servicios ecosistémicos que proporcionan los suelos según las categorías: de provisión, de regulación, de soporte y culturales. Esta consistió en cuatro etapas que posibilitaron evaluar la potencia de cada unidad cartográfica presente como la de la cuenca. En relación a los valores totales de los servicios ecosistémicos de las unidades cartográficas, se halló una diferencia del 5,8\% entre las de menor potencia con las de mayor potencia, indicando que los servicios ecosistémicos de estas últimas no son muy superiores a pesar de poseer mejores aptitudes productivas. Las de menor aptitud aportan atributos beneficiosos al ambiente que equilibran la cuenta total de las potencias de los eco-servicios que brindan. La proporción de los servicios ecosistémicos de regulación provistos por los suelos es la más alta (32\%), siendo menor los de soporte (17\%). En la cuenca estudiada, los suelos proporcionan un relevante aporte para el mantenimiento del equilibrio de funciones y procesos que resulta independiente de sus aptitudes productivas. Es el rol que cumplen como recurso que brinda beneficios múltiples, lo que permite reconocerlos como articuladores de varias dimensiones ambientales.
\end{abstract}

Palabras clave: suelos, servicios ecosistémicos, cuencas rurales

\begin{abstract}
Soils are part of the ecosystem and provide multiple services that are not properly considered by society. The objective of this work is to evaluate them in a rural basins, in order to identify and assess the environmental, social, cultural and economic functions and benefits they provide. In the Ludueña stream basin in the south of Santa Fe (Argentina), a methodology was applied to assess the power of the ecosystem services provided by the soils according to the categories: provision, regulation, support and cultural. This consisted of four stages that made it possible to evaluate the power of each cartographic unit present as well as of the basin. In relation to the total values of the ecosystem services of the cartographic units, a difference of 5.8\% was found between those with the lowest power and those with the highest power, indicating that the ecosystem services of the latter are not much superior despite possess better productive ability. Those of less aptitude contribute beneficial attributes to the environment that balance the total account of the powers of the eco-services they provide. The proportion of regulating ecosystem services provided by soils is the highest (32\%), with support being the lowest (17\%). In the studied basin, soils provide a relevant contribution to maintaining the balance of functions and processes that is independent of their productive aptitudes. It is the role they perform as a resource that provides multiple benefits, which allows them to be recognized as articulators of various environmental dimensions
\end{abstract}

Keywords: soils, ecosystem services, rural basins 


\section{INTRODUCCIÓN}

Las modificaciones producidas principalmente por el uso de la tierra en la agricultura, ha impactado en las capacidades que poseen los ecosistemas de brindar bienes y servicios que resultan de suma importancia para el desarrollo de las actividades humanas (Egoh et al., 2007; Metzger et al., 2006; Sarandón, 2014).

Los servicios que brindan los ecosistemas son beneficios múltiples que comprenden mejoras funcionales para los ambientes naturales y antropizados. Las cuencas representan espacios del territorio que reúnen las condiciones adecuadas para estudiar estos servicios. De acuerdo a lo establecido por Millennium Ecosystem Assessment (2005) se reconocen los siguientes tipos:

- Servicios de Abastecimiento: son los bienes y productos que se obtienen de los ecosistemas. Alimentos, fibra, combustible, bioquímicos, recursos genéticos y agua pura.

- Servicios de Regulación: son los beneficios resultantes de la regulación de los procesos ecosistémicos, inundaciones, control de plagas, polinización, dispersión de semillas, control de erosión, purificación de agua, control de enfermedades.

- Servicios Culturales: son los beneficios no materiales, valores espirituales y religiosos, sistema de conocimiento, educación, e inspiración, valores estéticos y recreativos.

- Servicios de Soporte: son aquellos necesarios para la producción de todos los otros servicios de los ecosistemas producción primaria, ciclo de nutrientes, provisión de hábitats, producción de oxígeno en atmósfera y agua.

Se asume que los suelos como parte de los ecosistemas proveen importantes servicios, y en este trabajo se planteó su evaluación en una cuenca agrohidrológica, con el propósito de identificar y valorar las funciones y beneficios ambientales, sociales, culturales y económicos que brindan.

La complejidad de los sistemas sociecológicos permite comprender la retroalimentación entre el sistema natural y el sistema social mediada por los servicios ecosistémicos y reflejada en los beneficios que suministran. La valoración integral de estos servicios también comprende la condición de incertidumbre y de sus variaciones espaciotemporales, y constituye una opción apropiada para abordarlos (Caro-Caro y Torres-
Mora, 2015). Esta integralidad permite además separar analíticamente el rol de los recursos naturales que conforman los ecosistemas y evaluar específicamente sus ecoservicios. El suelo es uno de ellos e influye considerablemente sobre el medio en el que se encuentra, condicionando las actividades sociales y económicas de los grupos humanos que se asientan en ese territorio, grupos que ejercen diferente grado de presión sobre el suelo y que generan su afectación o deterioro (Gardi et al., 2014).

El suelo es un componente clave para el bienestar del ecosistema y la salud del hombre y a pesar de ello, las políticas para protegerlo son todavía débiles, y no son de interés periodístico (López Ulloa, 2016), por esta razón es imprescindible intensificar las investigaciones que pongan en evidencia su relevancia.

El objetivo de este trabajo es evaluar los servicios ecosistémicos que brindan los suelos, con el propósito de identificar y valorar las funciones y beneficios ambientales, sociales, culturales $\mathrm{y}$ económicos, en la cuenca del arroyo Ludueña en el sur de Santa Fe (Argentina).

\section{MATERIALES Y MÉTODOS}

El estudio se realizó en la cuenca del arroyo Ludueña en la provincia de Santa $\mathrm{Fe}$, la cual posee una superficie de $83.418,3$ ha y se ubica entre los paralelos $32^{\circ} 45^{\prime}$ y $33^{\circ} 08^{\prime} \mathrm{S}$ y los meridianos $61^{\circ} 04^{\prime}$ y $60^{\circ} 39^{\prime} \mathrm{O}$ (Figura 1). El clima es subhúmedo mesotermal, con una temperatura media anual de $17{ }^{\circ} \mathrm{C}$ y con precipitaciones totales promedio de 1.110 mm (Cáceres, 1980).

Para el desarrollo del trabajo se plantearon cuatro etapas:

- Etapa 1: se identificaron las unidades cartográficas (UC) obtenidas del agrupamiento de suelos a escala 1:50.000 para la provincia de Santa Fe, digitalizadas y publicadas por la Infraestructura de Datos Espaciales (IDE) del Instituto Nacional de Tecnología Agropecuaria (INTA), Argentina (Nodo EEA Rafaela, S.F.). Las UC presentes en este sistema están definidas por Asociaciones y Consociaciones de suelos a nivel de serie, clasificados según el Sistema Soil Taxonomy (Soil Survey Staff, 1998). Las UC fueron obtenidas a partir de digitalización mediante escáner, georreferenciación a coordenadas globales y proyección, y posterior vectorización en pantalla de 
las unidades cartográficas que se corresponden con la cuenca del arroyo Ludueña, obrantes en la Carta de Suelos de la Rep. Argentina, en la Hoja 3360-14 (INTA, 1988). El software empleado fue QGIS 2.18.

- Etapa 2: se obtuvo la potencia de las series de suelo representativas de la cuenca para proveer los servicios (capacidad para realizarlo). Esta cuantificación se efectuó mediante la metodología elaborada por Castañeda Camacho (2013), la cual fue modificada y también aplicada en la misma cuenca por Montico et. al. (2019). El método contempla la utilización de criterios de evaluación y calificación de los mismos según se indica en la Tabla 1.

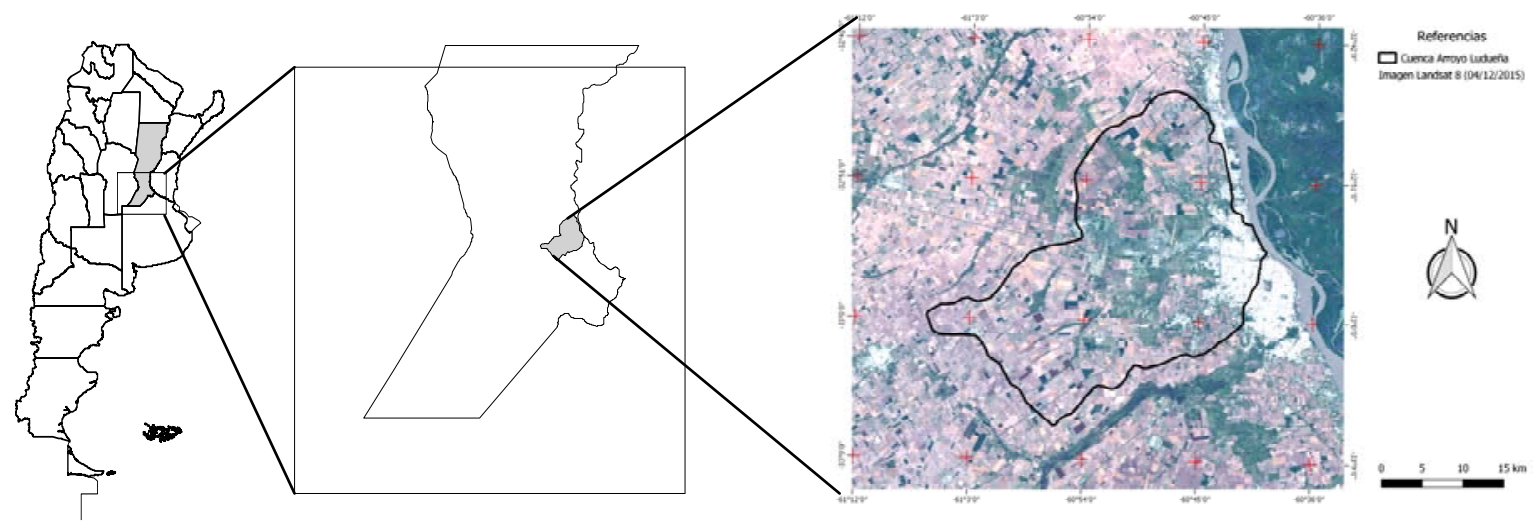

Figura 1. Ubicación de la cuenca en la provincia de Santa Fe.

Tabla 1. Descripción de los criterios de evaluación y valores de calificación utilizados en las series de suelo.

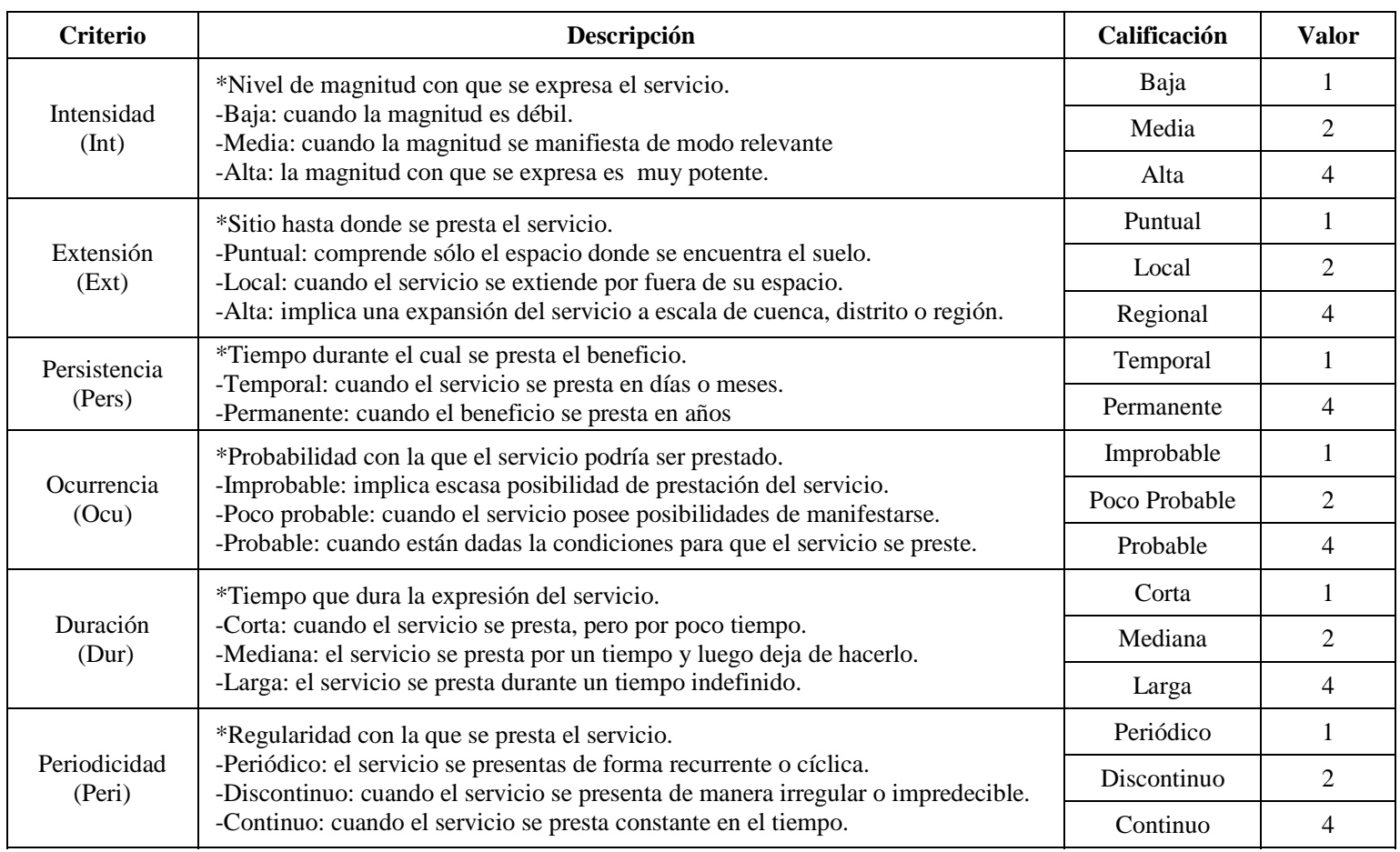

Para obtener la potencia de una serie de suelos, se serie de suelos se clasificó en categorías según se debe aplicar la ecuación (1). La potencia de cada indica en la Tabla 2.

Potencia $(\mathrm{P})=$ Intensidad $($ Int $)+$ Extensión $($ Ex $)+$ Persistencia $($ Pers $)+$ Ocurrencia $($ Ocu $)$ + Duración $($ Dur $)$ + Periodicidad $($ Peri $)$ 
Tabla 2. Valoración de la potencia de los eco-servicios que proveen las series de suelos.

\begin{tabular}{|c|c|}
\hline Calidad de la potencia del SE & Valor \\
\hline Muy Baja & $<5$ \\
\hline Baja & $5-9,9$ \\
\hline Media & $10-14,9$ \\
\hline Alta & $15-20$ \\
\hline Muy alta & $>20$ \\
\hline
\end{tabular}

- Etapa 3: se calculó la potencia de cada UC mediante la ponderación relativa de las potencias de las series edáficas en relación a la proporción en que participan en cada una de ellas.

- Etapa 4: mediante la sumatoria de las potencias de cada servicio ecosistémico de cada UC se obtuvo el valor total de los servicios ecosistémicos (VTSEuc) y a través de la sumatoria de las potencias de todas las UC se obtuvo el valor total de cada servicio ecosistémico en la cuenca (VTSEc).

\section{RESULTADOS Y DISCUSIÓN}

En la Tabla 3 se muestran las características más sobresalientes de las series de suelos de mayor representatividad areal en la cuenca.

Las UC que se indican en la Tabla 4, muestran la identidad de este recurso para proveer servicios ecosistémicos, dado que conjuntamente con lo descripto en la Tabla 3, aportan al reconocimiento de sus funciones y limitaciones.

La cartografía de las UC suelos de la cuenca se muestra en la Figura 2.

Tabla 3. Características de las series de suelo presentes en la cuenca Ludueña.

\begin{tabular}{|c|c|c|c|}
\hline Serie de suelo & Clasificación taxonómica & Familia & Drenaje \\
\hline Gelly & Natralbol típico & limosa fina, illítica, térmica & Imperfecto a pobre \\
\hline Monte Flores & Argialbol típico & arcillosa fina,illítica, térmica & Imperfecto \\
\hline Peyrano & Argiudol típico & arcillosa fina,illítica, térmica & Bueno \\
\hline Roldán & Argiudol típico/vértico & arcillosa fina,illítica, térmica & Bueno a moderado \\
\hline Zavalla & Natracualf típico & arcillosa fina, illítica, térmica & Pobre \\
\hline
\end{tabular}

Tabla 4. Representatividad, composición porcentual de las unidades cartográficas en la cuenca Ludueña, grupo de aptitud de tierra (GAT) e índice de aptitud de tierra (IAT) [Sistema de evaluación de tierras por aptitud de uso].

\begin{tabular}{|c|c|c|c|c|c|c|c|c|}
\hline Unidad cartográfica (UC) & Área & GAT & IAT & Gelly & Monte Flores & Peyrano & Roldán & Zavalla \\
\hline Py & 24,7 & 1 & 90 & & & 100 & & \\
\hline Py15 & 0,2 & 3/4ws & 49 & & & 100 & & \\
\hline Py20 & 1,0 & $2 \mathrm{w}$ & 75 & & & 100 & & \\
\hline Py2x & 1,7 & $2 \mathrm{w}(\mathrm{e})$ & 67 & & & 100 & & \\
\hline Py8 & 3,0 & $1(\mathrm{e})$ & 81 & & & 100 & & \\
\hline Rd & 9,3 & 1 & 82 & & & & 100 & \\
\hline Rd10 & 15,3 & $4 / 5 w s$ & 49 & 20 & 20 & & 30 & 30 \\
\hline Rd11 & 1,8 & $3 w(e)$ & 49 & 20 & 40 & & 40 & \\
\hline Rd12 & 15,9 & $2 / 3 w(p)$ & 72 & 10 & 20 & & 70 & \\
\hline Rd15 & 9,2 & $3 / 4 w s$ & 49 & 20 & 20 & & 50 & 10 \\
\hline Rd8 & 3,3 & $3 w s$ & 67 & 30 & & & 70 & \\
\hline Za2 & 2,2 & $7 w s$ & 8 & 20 & 30 & & & 50 \\
\hline Za3 & 8,3 & $5 w s$ & 22 & 20 & 30 & & & 50 \\
\hline
\end{tabular}




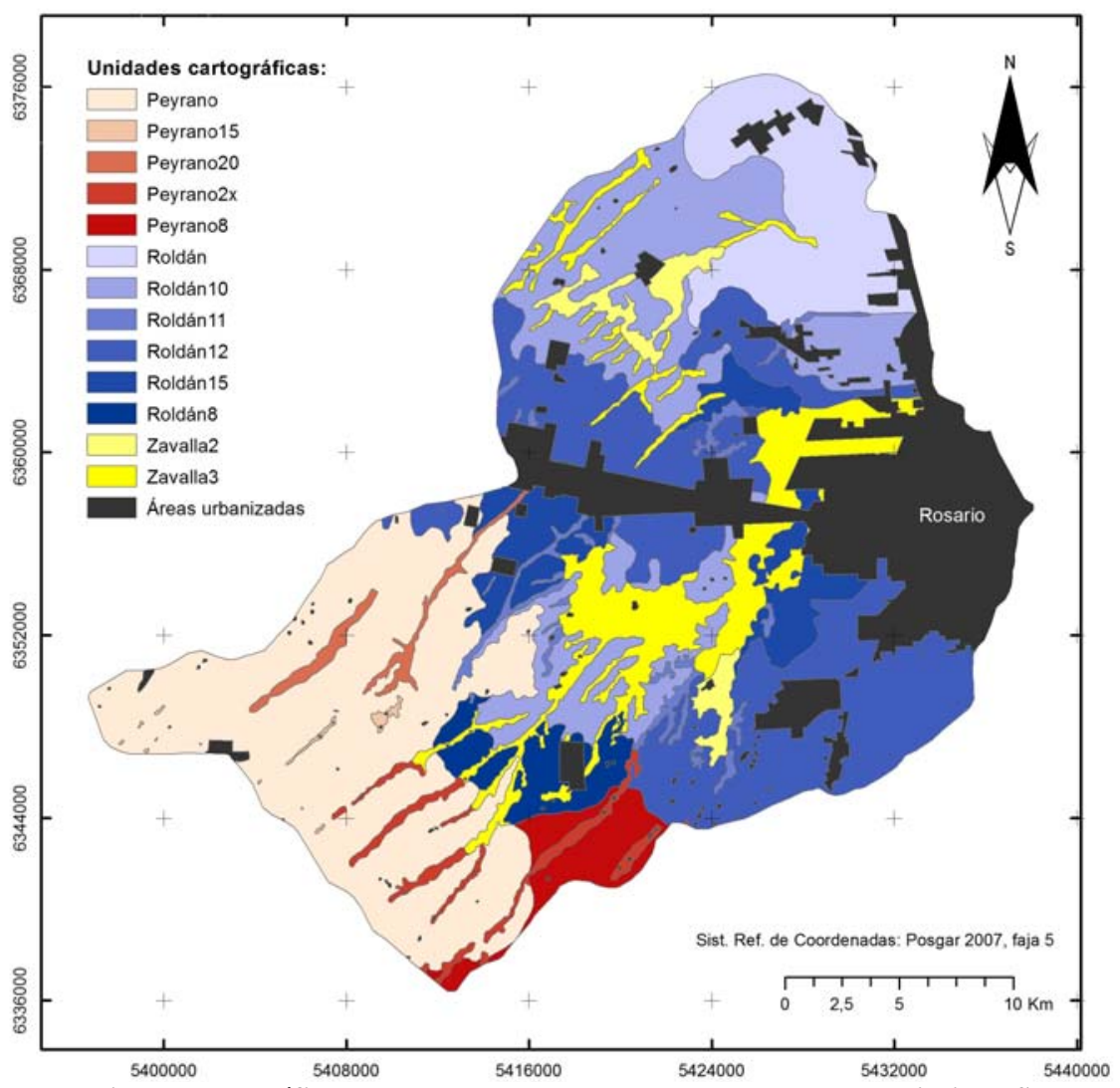

Figura 2. Mapa de unidades cartográficas presente en la cuenca del arroyo Ludueña, provincia de Santa Fe (Argentina).

Mediante los criterios de evaluación y valores de calificación de las series de suelo se obtuvo la potencia de cada una de ellas para proveer los diferentes servicios ecosistémicos (Tabla 5).

En la Tabla 6 se indican las potencias de los ecoservicios de las UC y los valores totales de la potencia de cada una de ellas (VTSEuc) y de cada servicio ecosistémico en la cuenca (VTSEc).

Del análisis de la matriz se destacan diferentes amplitudes de potencia entre las UC para los servicios ecosistémicos: 49 para los de Provisión, 4 para los de regulación, 15 para los de soporte y 26 para los culturales. La mayor amplitud en los de Provisión está asociada a las aptitudes productivas de la UC, las que por sus funciones $\mathrm{y}$ limitantes, principalmente edafogenéticas y ubicación paisajísticas, poseen mejores capacidades para sostener una producción amplia y diversificada de granos, oleaginosas, forrajes y forestales. La menor amplitud de los servicios de Regulación se debería a las compensaciones que se establecen entre las UC. Esto es, mientras para las que se encuentran en ambientes deprimidos, con pastizales naturales, cercanas a los escurrimientos y con napa freática próxima a la superficie, el aporte a plagas y enfermedades junto a corredor de biodiversidad es mayor, resulta menor en relación a las otras ubicadas en planos altos y con pendientes, sin napa cercana y con producción mayormente de cultivos de renta, donde es mayor el aporte a drenaje/inundación y a sumidero de carbono.

En relación a los VTSEuc, la diferencia del 5,8\% entre las UC de menor potencia (Za2 y Za3) con las de mayor potencia (Py, Py15; Py20, Py2x, Py8) indica que los servicios ecosistémicos de estas últimas no son muy superiores a pesar de poseer mejores aptitudes productivas. Precisamente, las de menor aptitud aportan atributos beneficiosos al ambiente que equilibran la cuenta total de las potencias de los ecoservicios que brindan. Esto fortalece la dimensión conceptual del servicio ecosistémico, dado que su relevancia no está regida por una valoración utilitaria basada sólo en las capacidades y fortalezas para producir comodities sino en las de proveer beneficios tangibles e intangibles que optimizan la calidad del ambiente y de la vida de las sociedades. Paruelo et al. (2006) reafirman este juicio cuando destacan las diferentes e importantes posibilidades de proveer ciertos servicios sin valor de cambio en el mercado frente a la producción de bienes comerciales como granos y oleaginosas, que poseen algunos componentes ambientales. 
Tabla 5. Matriz de potencia de los servicios ecosistémicos brindados por las series de suelo de la cuenca.

\begin{tabular}{|c|c|c|c|c|c|c|}
\hline & & & & & & \\
\hline & & \multicolumn{5}{|c|}{ Serie de suelo } \\
\hline & & Gelly & $\begin{array}{l}\text { Monte } \\
\text { Flores } \\
\end{array}$ & Peyrano & Roldán & Zavalla \\
\hline \multirow{4}{*}{$\begin{array}{l}\text { SERVICIOS DE } \\
\text { PROVISIÓN }\end{array}$} & Alimentos (Proteínas y Carbohidratos) & 7 & 7 & 24 & 24 & 6 \\
\hline & Madera & 14 & 16 & 24 & 24 & 10 \\
\hline & Fibras & 16 & 18 & 24 & 24 & 14 \\
\hline & Recursos genéticos & 12 & 12 & 24 & 24 & 12 \\
\hline \multirow{6}{*}{$\begin{array}{l}\text { SERVICIOS DE } \\
\text { REGULACION }\end{array}$} & Clima & 20 & 20 & 24 & 24 & 20 \\
\hline & Calidad superficial de agua & 13 & 13 & 13 & 13 & 13 \\
\hline & Drenaje/Inundación & 20 & 20 & 24 & 24 & 20 \\
\hline & Corredor de Biodiversidad & 20 & 20 & 12 & 12 & 20 \\
\hline & Sumidero de carbono & 18 & 18 & 24 & 24 & 18 \\
\hline & Plagas y enfermedades & 20 & 20 & 18 & 18 & 20 \\
\hline \multirow{3}{*}{$\begin{array}{l}\text { SERVICIOS DE } \\
\text { SOPORTE }\end{array}$} & Intervención en los ciclos biogeoquímicos & 20 & 20 & 20 & 20 & 20 \\
\hline & Provisión de hábitats & 24 & 24 & 11 & 11 & 24 \\
\hline & Intervención en el ciclo hidrológico & 24 & 24 & 24 & 24 & 24 \\
\hline \multirow{5}{*}{$\begin{array}{l}\text { SERVICIOS } \\
\text { CULTURALES }\end{array}$} & Recreativo & 24 & 24 & 14 & 11 & 24 \\
\hline & Estético & 24 & 24 & 14 & 11 & 24 \\
\hline & Educativo & 20 & 20 & 20 & 20 & 20 \\
\hline & Identidad del sitio & 24 & 24 & 24 & 24 & 24 \\
\hline & Herencia cultural & 24 & 24 & 24 & 24 & 24 \\
\hline
\end{tabular}

Tabla 6. Potencia de los eco-servicios provistos por cada unidad cartográfica y valores totales de las potencias de la unidades cartográficas y de la cuenca.

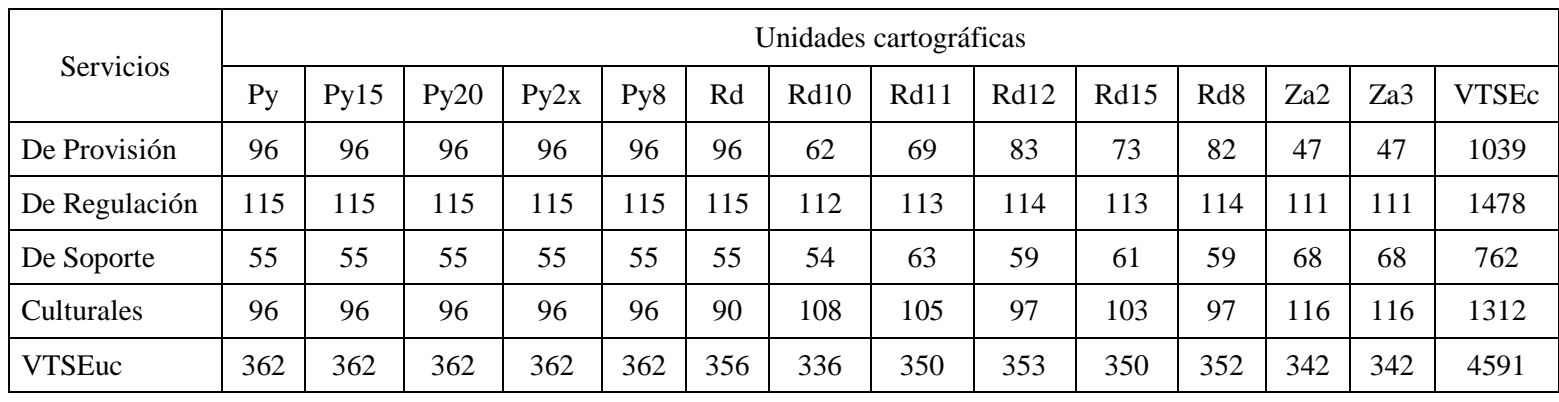

Tal como se asevera en un estudio realizado en la cuenca sobre las diferentes coberturas (Montico et al., 2019), la restricción al suministro de mayores servicios ecosistémicos también depende de la extensión areal del componente que lo provee, es decir, aunque se destaque por su provisión, la baja representatividad superficial en la cuenca condiciona su aporte global.

De la Tabla 6 se deduce que existe una importante diferencia de potencia entre los VTSEc. Tal como se muestra en la Figura 3, la proporción de los servicios ecosistémicos de regulación provistos por los suelos es la más alta (32\%), siendo menor los de soporte.

Esta condición jerarquiza la capacidad del recurso por intervenir en funciones $y$ procesos que interactúan con el clima, el drenaje, el control de plagas y enfermedades, el sostenimiento de la biodiversidad, el almacenaje de carbono y la calidad del agua. Además se destacan los servicios culturales que brindan los suelos por sobre los de provisión. Esto indica que a pesar del reconocimiento que poseen los suelos como sostén 
de la producción de alimentos, fibras y madera, es mayor el que tienen cuando se consideran los valores no materiales, es decir, aquellos vinculados a la significancia humanística.

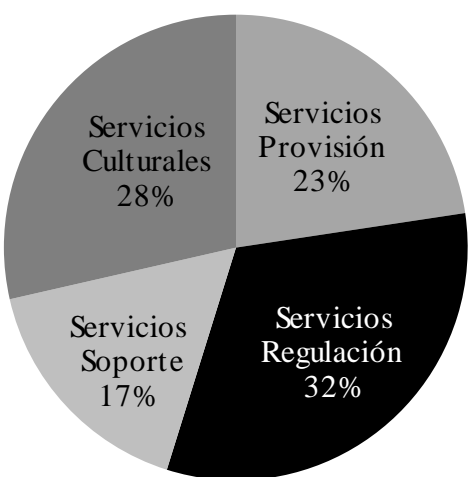

Figura 3. Proporción de los servicios ecosistémicos provistos por la cuenca.

Los suelos de la cuenca se erigen como pilares del sostenimiento de los servicios ecosistémicos y resulta necesario que este atributo sea reconocido por la sociedad más allá del proceso de generación de renta en el que participan. Las herramientas metodológicas que permitan evaluar su aporte pueden respaldar esta aseveración y fortalecer la consideración social (Rositano y Ferraro, 2017).

Según Camacho Valdez y Ruiz Luna (2012), la mayoría de las clasificaciones de los servicios ecosistémicos tienen como propósito principal mantener la salud de los ecosistemas y garantizar la provisión de sus servicios, pero también lo es considerar el contexto social y político dentro del cual los servicios ecosistémicos van a ser evaluados o utilizados. Así, entonces, la valoración de los suelos como proveedores de eco-servicios debe contextualizarse a nivel territorial comprendiendo todas las dimensiones ambientales involucradas. Tal como plantea Maris (2012), es imprescindible que la sociedad tenga un mayor involucramiento en las cuestiones vinculadas al sostenimiento de los beneficios que otorga la naturaleza.

\section{CONCLUSIONES}

En la cuenca estudiada, los suelos proporcionan un relevante aporte para el mantenimiento del equilibrio de funciones $\mathrm{y}$ procesos que resulta independiente de sus aptitudes productivas. Es el rol que cumplen como recurso que brinda beneficios múltiples lo que permite reconocerlos como articuladores de varias dimensiones ambientales. La recuperación y restauración de suelos, tanto como su preservación y conservación, es un imperativo de la sustentabilidad ambiental, sea cual fuere su capacidad productiva, la cual es una valoración insuficiente al momento de evaluar su relevancia ambiental.

\section{REFERENCIAS}

Cáceres, L. M. (1980). Caracterización climática de la provincia de Santa Fe. Ministerio de Agricultura y Ganadería. Provincia de Santa Fe, Argentina. 35p.

Camacho Valdez, V. y Ruiz Luna, A. (2012). Marco conceptual y clasificación de los servicios ecosistémicos. Revista Bio Ciencias 1 (4), 3 - 15.

Caro-Caro, C. I. y Torres-Mora, M. A. (2015). Servicios ecosistémicos como soporte para la gestión de sistemas socioecológicos: aplicación en agroecosistemas. Orinoquia, 19 (2), 237-252.

Castañeda Camacho, A C. (2013). Diseño de una metodología para evaluar el estado de los servicios ecosistémicos. Bogotá: Universidad Militar Nueva Granada - especialización en planeación ambiental y gestión integral de los recursos naturales.

Egoh, B.; Rouget, M.; Reyers, B.; Knight, A T.; Cowling, M R.; van Jaarsveld, A S. y Welz, A. (2007). Integrating ecosystem services into conservation assessments: a review. Ecol. Econ. 63, 714-721.

Gardi, C.; Angelini, M.; Barceló, S.; Comerma, J.; Cruz Gaistardo, C.; Encina, A.; Jones, A.; Krasilnikov, P.; Mendonça, M.; Montanarella, L.; Muñiz, O.; Schad, P.; Vara, M. y Vargas, R. (2014). Atlas de suelos de América Latina y el Caribe. Comisión Europea - Oficina de Publicaciones de la Unión Europea, L-2995, Luxembourg, 176 p.

Instituto Nacional de Tecnología Agropecuaria INTA (1988). Carta de Suelos de la República Argentina. Hoja 3560-13 y 14 "Cañada de Gómez” y “Rosario” Escala 1:50000. 197 p.

López Ulloa, R. M. (2016). Servicios ecosistémicos del suelo Revista Científica Ecuatoriana. 10-12.

Maris, V. (2012). De la naturaleza a los servicios ecosistémicos - una mercantilización de la biodiversidad. Ecología Política, 44, 27-32.

Metzger, M. J.; Rounsevell, M. D. A.; AcostaMichlik, L.; Leemans, R. y Schroter, D. (2006). The vulnerability of ecosystem services to land use change. Agriculture, Ecosystems and Environment 
114, 69-85.

Millennium Ecosystem Assessment. (2005). Ecosystems and Human Well-being: Synthesis. Washington D.C.: Island Press.

Montico, S; Di Leo, N; Bonel, B. y Denoia, J. (2019). Cambios del uso de la tierra en la cuenca del arroyo Ludueña, Santa Fe: impacto en la sostenibilidad y en los servicios ecosistémicos. Cuadernos del CURIHAM, 25, 31:39. ISSN 15142906 impresa, ISSN 2683-8168, en línea.

Paruelo, J. M.; Guerschman, J. P.; Piñeiro, G.; Jobbágy, E. G.;Verón, S. R.; Baldi, G. y Baeza, S. (2006). Cambios en el uso de la tierra en Argentina y Uruguay: marcos conceptuales para su análisis. Agrociencia, X(2), 47-61.

Rositano, F. y Ferraro, D.O. (2017). Una nueva aproximación metodológica basada en redes conceptuales y redes probabilísticas para evaluar la provisión de servicios de los ecosistemas. Ecología Austral, 27 (1).

Sarandón, S. (2014). El agroecosistema: Un ecosistema modificado. En: Sarandón, S. y Flores, C. (Eds.) Agroecología. Bases teóricas para el diseño y manejo de agroecosistemas sustentables.
La Plata, Buenos Aires, Argentina: Universidad Nacional de La Plata, 100-130.

Soil Survey Staff. (1998). Keys to soil taxonomy. 8th Edition,US Govt. Printing Office, Washington, DC, USA. 305 p.

\section{Tipo de Publicación: ARTícULO.}

Trabajo recibido el 10/01/2021 y aprobado para su publicación el 25/04/2021.

\section{COMO CITAR}

Montico, S.; Di Leo, N.; Bonel, B. y Berardi, J. (2021). Servicios Ecosistémicos provistos por los suelos en una cuenca de Santa Fe, Argentina. Cuadernos del CURIHAM, 27, 1-8. DOI: https://doi.org/10.35305/curiham.v27i.166

Este es un artículo de acceso abierto bajo licencia: Creative Commons Atribución - No Comercial Compartir Igual 4.0 Internacional (CC BY-NC-SA 4.0) (https://creativecommons.org/licenses/by-ncsa/4.0/deed.es) 